\title{
ASTEROID SPECTROSCOPY AND MINERALOGY
}

\author{
THOMAS H. BURBINE AND RICHARD P. BINZEL \\ Department of Earth, Atmospheric and Planetary Sciences, MIT, \\ Cambridge, MA 02139, U.S.A. \\ E-mail THB@ASTRON.MIT.EDU \\ E-mail RPB@ASTRON.MIT.EDU
}

\begin{abstract}
Spectroscopic observations of asteroids of smaller sizes and in the nearinfrared have led to many exciting discoveries that have increased substantially our knowledge of the mineralogy of asteroids. These discoveries include the identification of hydrated $\mathrm{M}$ and $\mathrm{E}$-asteroids, the mineralogical diversity of the S-class, anomalous $\mathrm{S}$ asteroids, a possible source body of the aubrites, Vesta-like objects in the main belt, possible ordinary chondrite-like objects and one of the reddest objects in the solar system.
\end{abstract}

\section{Introduction}

Since the publication of Asteroids II in 1989, there has been a considerable amount of work in asteroid spectroscopy that has increased substantially our knowledge of the mineralogical diversity of asteroids within and beyond the asteroid belt. Asteroid types that were just postulated to exist have been discovered in the past few years. These discoveries coupled with the identification of some objects not even considered to exist have led to a very exciting time in asteroid spectroscopy. This review paper will discuss seven of these discoveries and they include

1) the hydrated $M$ and E-asteroids,

2) the mineralogical diversity of the S-class,

3) the two anomalous S-asteroids (387 Aquitania and 980 Anacostia) with features uncharacteristic of any other type of S-asteroid,

4) the linkage of the earth-crossing E-asteroid 3103 Eger with the aubrites,

5) the Vesta-like objects in the Vesta family and also extending out to the Jovian 3 : 1 resonance region,

6) a possible ordinary chondrite-like object in the main belt and

7) the outer solar system asteroid 5145 Pholus that is one of the reddest objects in the solar system.

In addition to this paper, readers should also refer to more extensive recent reviews by Gaffey et al. (1993b) and Pieters and McFadden (1994).

\section{Asteroid Taxonomy and Mineralogy}

The most widely used taxonomy today is the one developed by Tholen (1984) using seven colors (normalized to the visual filter) from the Eight-Color Asteroid Survey (ECAS) (Zellner et al. 1985), which observed 589 asteroids using eight broadband filters from 0.34 to $1.04 \mu \mathrm{m}$. Tholen created fourteen asteroid classes with eleven of the classes distinguished by their ECAS spectra alone. Three classes ( $\mathrm{E}, \mathrm{M}$ and P) had degenerate ECAS spectra and could only be distinguished by visual albedo. Later taxonomies by Barucci et al. (1987) and Tedesco et al. (1989) used different 
classification methods on the ECAS data set in conjunction with IRAS albedos. The resulting taxonomies that they produced yielded classifications similar to Tholen's.

Mineralogical analysis of an asteroid's spectrum is done by comparing it to the spectral characteristics of laboratory measured materials. [Detailed reviews of asteroid and mineral spectroscopy can be found in Gaffey et al. (1989) and Gaffey et al. (1993b).] The mineralogical information that can be obtained from the analysis of a visual wavelength spectrum is the presence or absence of an ultraviolet absorption feature due to $\mathrm{Fe}^{+2}$, the presence or absence of an absorption feature due to olivine and/or pyroxene that appears longward of $0.7 \mu \mathrm{m}$ and is centered approximately between 0.9 and $1.1 \mu \mathrm{m}$ (usually called the $1 \mu \mathrm{m}$ feature) and the slope of the spectrum longwards of $0.55 \mu \mathrm{m}$ that is due to presence or absence of some reddening agent (e.g., organics, $\mathrm{NiFe}$ ). Also the visual albedo can be used to discriminate between different mineralogical types that have similar visual spectral characteristics. Mineralogical analysis of ECAS spectra is hampered by the low resolution of the spectra with reflectances at only eight wavelengths from 0.3 to $1.1 \mu \mathrm{m}$, the coverage of only part of the $1 \mu \mathrm{m}$ absorption feature (when present) that can extend out into the near-infrared and the lack of any information on the presence or absence of a $2 \mu \mathrm{m}$ absorption feature usually due to pyroxene.

The analysis of the ECAS data, in conjunction with a number of other data sets (e.g., radar, near-infrared photometry) that were done for much smaller numbers of asteroids, led to a picture of the asteroid belt up to 1989 that is best described by the Bell superclass paradigm (Bell 1986, Bell et al. 1989) (Table I). This paradigm groups the asteroid classes according to the degree of metamorphic heating that they are believed to have received. A very general mineralogic interpretation is given for each class plus the meteorites that are possible analogs to asteroids in that class. However the seven discoveries that are discussed in this paper will show that many of the asteroid classes are now known to contain very mineralogically diverse assemblages and that Table I gives only a very general framework of the asteroid belt. Recent revisions of the Bell superclass paradigm have been done by Burbine and Bell (1993a, 1993b) to try to better represent the compositional diversity within the classes.

\section{Asteroid Spectroscopy}

In addition to the progress in mineralogic analyses, substantial advances have been made in the observational techniques for asteroid spectroscopy at both visual and near-infrared wavelengths. The primary factor in these advances has been the incorporation of charge-coupled devices (CCDs) into asteroid spectroscopic observing programs. Because CCDs are area detectors with high quantum efficiencies, complete visual spectra within all or part of the range from 0.4 to $1.0 \mu \mathrm{m}$ can be recorded in a single exposure with a much higher spectral resolution $(\sim 10 \AA)$ than filter photometry. Simultaneous recording of all parts of the spectrum (as well as the sky) makes the measurements insensitive to flux variations caused by the irregular shape of a rotating asteroid. At visual wavelengths, several researchers have led the movement into using CCDs for asteroid spectroscopy (e.g., Luu and Jewitt 1990, Vilas and McFadden 1993). Improved quantum efficiencies at near-infrared 
TABLE I

Bell Superclasses

\begin{tabular}{|c|c|c|c|}
\hline Superclass & Class & Inferred Minerals & Analogous Meteorites \\
\hline Primitive & $\begin{array}{l}\text { D } \\
\text { P } \\
\text { C } \\
\text { K }\end{array}$ & $\begin{array}{r}\text { clays, organics } \\
\text { clays, organics } \\
\text { clays, C, organics } \\
\text { ol, pyx, carbon }\end{array}$ & $\begin{array}{r}\text { (none) } \\
\text { (none) } \\
\mathrm{CI}, \mathrm{CM} \text { chondrites } \\
\mathrm{CV} \text { and } \mathrm{CO} \text { chondrites }\end{array}$ \\
\hline Metamorphic & $\begin{array}{r}\mathrm{T} \\
\mathrm{B}+\mathrm{G}+\mathrm{F} \\
\mathrm{Q}\end{array}$ & $\begin{array}{r}? \\
\text { clays, opaques } \\
\text { pyx, ol, gray NiFe }\end{array}$ & $\begin{array}{l}? \\
\text { altered carb. chondrites } \\
\text { H, L, LL chondrites? }\end{array}$ \\
\hline Igneous & $\begin{array}{l}\text { V } \\
\text { R } \\
\text { S } \\
\text { A } \\
\text { M } \\
\text { E }\end{array}$ & $\begin{array}{r}\text { plag, pyx, ol } \\
\text { ol, pyx } \\
\text { pyx, ol, red NiFe } \\
\text { ol } \\
\mathrm{NiFe} \\
\text { Fe-free pyx }\end{array}$ & $\begin{array}{r}\text { basaltic achondrites } \\
\text { ol-rich achondrites? } \\
\text { pallasites, lodranites, irons ? } \\
\text { brachinites } \\
\text { irons } \\
\text { aubrites }\end{array}$ \\
\hline
\end{tabular}

wavelengths have allowed some important asteroid discoveries (discussed below) and have facilitated observations being pushed to fainter and smaller asteroids (e.g., Clark 1993).

The Small Main-Belt Asteroid Spectroscopic Survey (SMASS), initiated at the Massachusetts Institute of Technology in 1990 (Binzel and Xu 1990, Xu et al. 1993, $\mathrm{Xu}$ 1994) provides an example of the potential for asteroid spectroscopy using CCDs. Figure 1 shows the approximate size limits for asteroids that were wellsampled by previous surveys, such as the Eight-Color Asteroid Survey by Zellner et al. (1985). Use of CCDs with modern spectrographs on two-meter telescopes allows the limit for visual spectra to be pushed to well below $10 \mathrm{~km}$ diameter asteroids in the inner main-belt. The effective limit for SMASS, which utilizes the $2.4 \mathrm{~m}$ Hiltner telescope of the Michigan-Dartmouth-MIT Observatory, is shown in Figure 1. This new capability to spectroscopically sample nearly all of the numbered asteroids has lead to several important discoveries (discussed below) and promises a tremendous potential for further advances during the 1990s.

Another important observational technique is rotationally resolved spectroscopy where spectra are taken at different times throughout the asteroid's rotational period. These rotationally resolved spectra allow for compositional differences to be measured across an asteroid. This technique has been used to make a compositional map of Vesta (Gaffey 1983), to determine the igneous nature of 8 Flora (Gaffey 1984 ) and to characterize compositional differences across 243 Ida (Barucci and Lazzarin 1993). 


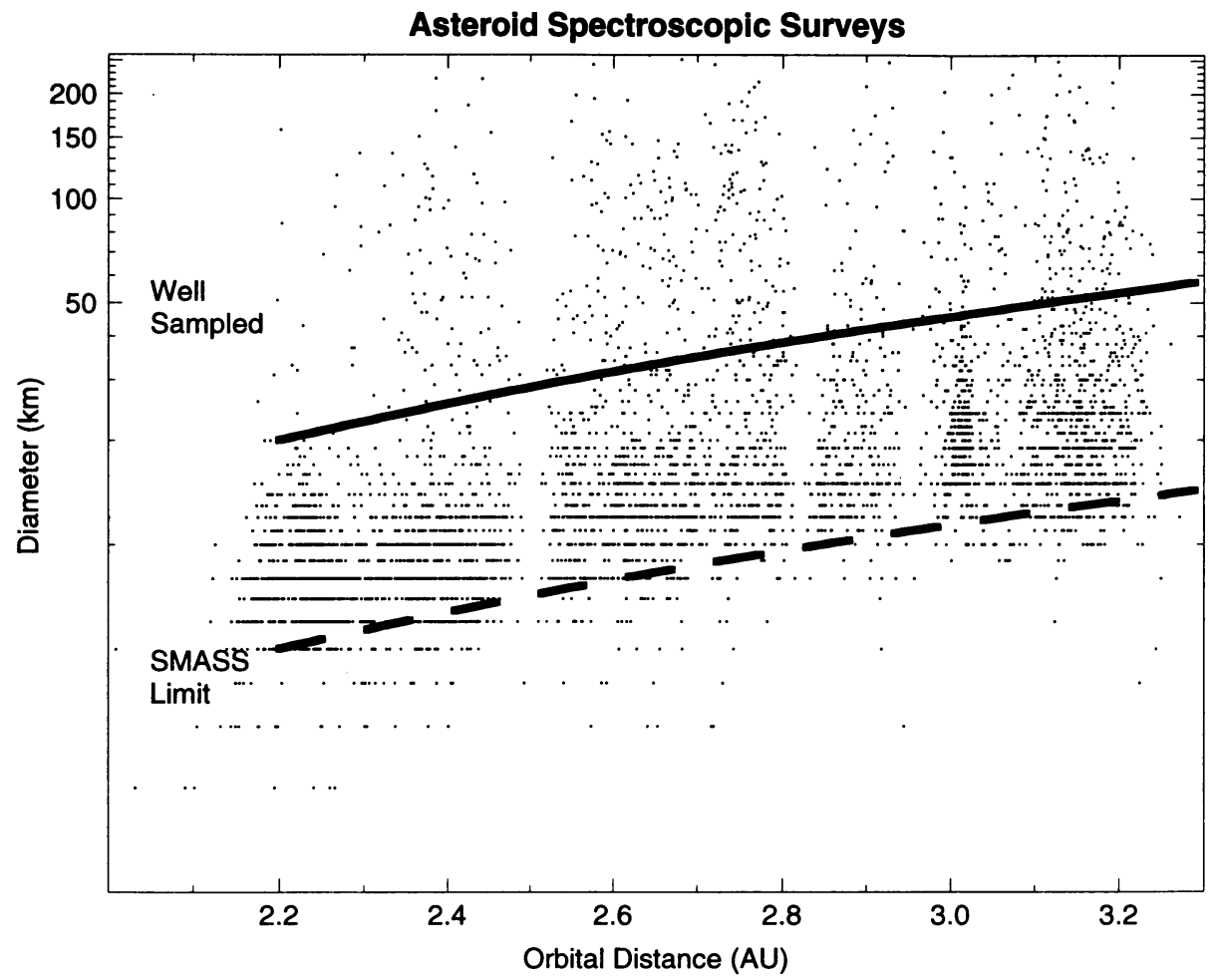

Fig. 1. Orbital distribution of asteroids plotted versus their diameters. The top line depicts the approximate limit where previous surveys have obtained reasonable sampling statistics among the larger asteroids. With the advent of CCDs, programs such as the Small Main-Belt Asteroid Spectroscopic Survey (SMASS, lower dashed line) can routinely sample the spectral properties of main-belt asteroids having diameters of about $10 \mathrm{~km}$ or less.

\section{Hydrated $M$ and E-asteroids}

Spectra from approximately 2.5 to $3.5 \mu \mathrm{m}$ allow the determination of the presence 
or absence of a broad absorption feature centered at approximately $3 \mu \mathrm{m}$ that is due to hydrated silicates on the surface of an asteroid. The presence of hydrated silicates indicates that the original parent bodies, believed to have been composed of anhydrous silicates and ice, underwent aqueous alteration due to a mild heating episode that raised temperatures in the interior above the freezing point of water. The most detailed observational work in this area was done by Jones et al. (1990), who analyzed $3 \mu \mathrm{m}$ observations of 46 asteroids. They found that approximately two-thirds of the C-type asteroids had hydrated silicate surface compositions.

Observations were also done on "igneous" asteroids (asteroids whose surface mineralogies are believed to have formed from a melt) to try to confirm that $3 \mu \mathrm{m} \mathrm{ab}-$ sorption features are not characteristic of these supposedly "anhydrous" asteroids. Observations of three S-asteroids and 4 Vesta confirmed the absence of hydrated silicates on the surfaces of these asteroids. However two of the three M-class asteroids observed (55 Pandora and 92 Undina) were found to have $3 \mu \mathrm{m}$ features that seem to indicate the presence of hydrated silicates and not the metallic iron compositions that are the mnemonic corresponding to the classification letter "M." [Undina was originally given the designation of X (EMP) by Tholen (1984) due to the asteroid's lack of a visual albedo at that time, but was given the designation of $\mathrm{M}$ based on its IRAS albedo of 0.20 in other taxonomies (Barucci et al. 1987, Tedesco et al. 1989).] The $3 \mu \mathrm{m}$ observations of these two asteroids have been repeated to try to confirm or reject the presence of hydrated silicates (Howell personal communication, Rivkin et al. 1994). Preliminary observations of 55 Pandora (Howell personal communication) are not consistent with the observations of Jones et al. (1990) and appear to indicate an anhydrous assemblage. However observations of 92 Undina (Rivkin et al. 1994) confirm the presence of hydrated silicates on this asteroid. The observed M-asteroid without a $3 \mu \mathrm{m}$ feature is 16 Psyche, which is generally thought to have a considerable metallic iron component due to its high radar albedo (Ostro et al. 1985).

Observations were also made of the E-type asteroid 44 Nysa. Nysa and other E-type asteroids have been thought to have surfaces dominated by a nonabsorbing, spectrally neutral phase such as enstatite, forsterite or some other iron-poor silicate due to their high albedos and relatively featureless spectra (Gaffey et al. 1989). These asteroids are believed to have surfaces that are the crusts or exposed mantles of differentiated parent bodies with E-chondrite bulk compositions (Zellner et al. 1977). Nysa also has a weak $0.85-0.90 \mu \mathrm{m}$ pyroxene feature, not evident in other E-type asteroid spectra, that is consistent with low-iron enstatite seen in some meteorites (Gaffey et al. 1989). However $3 \mu \mathrm{m}$ observations of Nysa (Howell personal communication, Rivkin et al. 1994) appear to indicate the presence of hydrated silicates on this asteroid. Either the E or M-types with $3 \mu \mathrm{m}$ features are not igneous or some currently unknown post-igneous aqueous alteration processes have operated on these asteroids. Thus, these observations appear to indicate that some percentage of the classified $\mathrm{M}$ and E-asteroids may have compositions similar to the C-type asteroids. Just as importantly, the apparent discovery of hydrated silicates among asteroids classified as " $M$ " and " $E$ " reminds us that taxonomy is based on the characteristics of spectral features and does not automatically carry with it an interpretation of the mineralogy. 


\section{Diversity of the S-class}

High-resolution spectra from 0.8 to $2.5 \mu \mathrm{m}$ allow for a better determination of the $1 \mu \mathrm{m}$ feature, the degree to which the spectrum is reddened, the identification of the presence or absence of absorption features such as the $1.1 \mu \mathrm{m}$ feature due to feldspar, a $2 \mu \mathrm{m}$ feature (due to minerals such as pyroxene and spinel) and a 2.2 $\mu \mathrm{m}$ feature attributed to organics (Cruikshank et al. 1991a). The most extensive observing program in this wavelength range was done by Bell et al. (1988) who observed 119 asteroids (predominately of the S-class) as part of the 52-channel (also known as the 52-color) survey. Analyses of the 52-channel data have led to the creation of the K-class (Bell 1988), which are objects with S-like ECAS spectra but flat reflectances in the near-infrared, and the creation of a preliminary asteroid taxonomy using a neural network (an artificial intelligence program that will recognize patterns in a data set) (Howell et al. 1994). This taxonomy supports the separation of the $\mathrm{K}$-class from the $\mathrm{S}$-class and also defines two sub-classes within the S-class (So and Sp). So-asteroids are olivine-rich with a red continuum slope while $\mathrm{Sp}$-asteroids are pyroxene-rich with a less red continuum slope. The remaining S-asteroids show intermediate olivine-pyroxene compositions with a moderately red continuum slope.

The 52-channel data, in conjunction with ECAS and/or 24-filter data, yield coverage of both the 1 and $2 \mu \mathrm{m}$ absorption features thereby allowing much better mineralogical analyses of asteroids (A, S and V-classes) that have features predominately due to olivine and/or pyroxene. From the initial classification by Tholen (1984), the S-asteroids were known to be a very compositionally diverse spectral class, but no subdivision of the S-class was attempted. The spectral range and resolution of the 52-channel data allow for a much better determination of the mineralogic characteristics of the olivine and/or pyroxene absorption features that appear to be crucial for sub-dividing the S-class. For olivine-orthopyroxene mixtures, the relative areas of the 1 and $2 \mu \mathrm{m}$ features (called the band area ratio) and the center of the $1 \mu \mathrm{m}$ feature (called the band I center) have been found to be related to the olivine-orthopyroxene abundance (Cloutis et al. 1986). Gaffey et al. (1993a) used these parameters to group 39 S-asteroids with 52-channel spectra into seven compositionally distinct subtypes [S(I)-S(VII)]. The reflectance spectra of S(I)-asteroids are indicative of monomineralic olivine assemblages, the spectra of the $\mathrm{S}(\mathrm{II})$ to $\mathrm{S}(\mathrm{VI})$-asteroids are indicative of varying olivine/pyroxene abundances and the spectra of S(VII)-asteroids are indicative of pyroxene or pyroxene- feldspar assemblages.

These spectral parameters (band area ratio and band I center) were also compared to the spectral parameters derived from the analysis of the spectra of ordinary chondrites. Except for the S(IV)-subclass, the S-asteroids have very different spectral parameters from the ordinary chondrites, which implies different silicate (olivine and pyroxene) mineralogies and no relationship between these two sets of objects. The S(IV)-asteroids (e.g., 3 Juno, 6 Hebe, 7 Iris) have silicate mineralogies consistent with ordinary chondrites and appear to be the only viable ordinary chondrite parent body candidates among the large main-belt S-asteroid population. However the silicate mineralogies of the S(IV)-asteroids are also consistent with the 
mineralogy of objects that have undergone small degrees of partial differentiation (primitive achondrites), so these objects need to be more intensely studied before any definite conclusions can be made. It is interesting to note that the S(IV)asteroids appear to cluster at semimajor axes between 2.3 and 2.6 AU (Gaffey et al. 1993a), just where the Jovian $3: 1$ and $\nu_{6}$ secular resonances both provide viable dynamical routes to Earth-crossing orbits (e.g., Farinella et al. 1993).

\section{Anomalous S-asteroids}

The 52-channel data also have allowed for the identification of some asteroids that appear distinctly different when compared to other S-types. Burbine et al. (1992) identified S-asteroid 387 Aquitania and SU-asteroid 980 Anacostia as having absorption features not characteristic of assemblages of olivine and/or pyroxene. The reflectance spectra of assemblages of olivine and/or pyroxene always have a $1 \mu \mathrm{m}$ feature stronger than the corresponding $2 \mu \mathrm{m}$ feature. In contrast, these two asteroids each have a $2 \mu \mathrm{m}$ feature stronger than the corresponding weak to non-existent 1 $\mu \mathrm{m}$ feature. This feature has been interpreted as being either due to spinel (Burbine et al. 1992) or chromite (a member of the spinel group) (Cloutis and Gaffey 1993). The presence of spinel would appear to indicate a surface composed of $\mathrm{CO} / \mathrm{CV}$ carbonaceous material enriched in calcium-aluminum (CAI) inclusions while the presence of chromite would indicate an achondritic surface enriched in chromite that maybe similar to the composition of two unique chromite-rich achondrites that have just been recently described (Meteorite Working Group 1993, Takeda et al. 1993).

\section{E-asteroid 3103 Eger and the aubrites}

As stated before, E-asteroids, because of their high albedos and relatively featureless spectra, have been interpreted as having surfaces dominated by a nonabsorbing, spectrally neutral phase such as enstatite or forsterite. These E-asteroids have been thought to be the probable parent bodies of the aubrites or enstatite achondrites (e.g., Zellner et al. 1977). The Hungaria asteroid region, which is located between $1.79 \mathrm{AU}$ and $1.98 \mathrm{AU}$, is dominated by E-types and contains approximately half of all E-types known in the asteroid belt.

The only Earth-crossing E-type asteroid known is 3103 Eger (provisionally designated as $1982 \mathrm{BB}$ ), which was classified as an E-type (Veeder et al. 1989) due to its relatively neutral visual colors (Wisniewski 1987) and high albedo (0.530.63) (Veeder et al. 1989). Observations by Gaffey et al. (1992) in the visible and near-infrared confirmed that Eger has a featureless spectrum consistent with its classification as an E-type. Gaffey et al. (1992) have proposed that this object is the actual near-Earth parent body of the aubrites. The lines of evidence include the rareness of E-type asteroids in Earth-approaching orbits, the time of day of aubrite falls that indicates a limited range of source orbits with many of those orbits similar to Eger, the clustering of cosmic ray exposure ages (which records the time that a meteorite spent within a meter of the surface of its original parent body or as a meter-size fragment in interstellar space) for the aubrites and similarities in mineral compositions that suggest that most aubrites are derived from a single parent 
body (e.g., Keil 1989). The aphelion distance of Eger (1.905 AU) falls within the Hungaria zone and this fact, in conjunction with this asteroid's designation as an E-type, appears to indicate that Eger was most probably derived from that region. These results represented the first plausible direct link between a meteorite group (aubrites) and a particular source region of the asteroid belt.

\section{Vesta-like Asteroids}

Asteroid 4 Vesta is unique in being the only large main-belt asteroid in the Vclass. Vesta's reflectance spectrum also is known to be very similar to the spectra of basaltic achondritic meteorites (McCord et al. 1970, Feierberg et al. 1980, Feierberg and Drake 1980). This spectral similarity argued for Vesta being the source of the basaltic achondrites, however cratering mechanics argued against Vesta being the source due to the belief (Wetherill 1987) that ejection velocities would not be high enough to allow fragments to reach some of the dynamically favored meteorite source regions such as the Jovian $3: 1$ and $\nu_{6}$ secular resonances. But the identification of a statistically robust family around Vesta (Zappalá et al. 1990) showed that fragments could be ejected from Vesta's surface at velocities well exceeding the escape velocity $(300 \mathrm{~m} / \mathrm{s})$ and could obtain velocities $(>600 \mathrm{~m} / \mathrm{s})$ that were high enough to reach the Jovian $3: 1$ resonance (Farinella et al. 1993). Three basaltic Earth approaching asteroids were also found to exist (Cruikshank et al. 1991b), however it was still very difficult to persuasively argue that Vesta was the source of the basaltic achondrites.

Recent CCD spectroscopy of small asteroids (diameters $\leq 10 \mathrm{~km}$ ) near Vesta appears to have established Vesta as indeed being the source of the basaltic achondrite meteorites. These asteroids were part of a spectroscopic survey of Xu (1994) whose goal was to determine the spectral characteristics of these small main-belt asteroids so as to reveal possible difference in composition between the large and small asteroid populations and hopefully establish new links in the asteroid-meteorite connection. Binzel and Xu (1993) identified twenty-one main-belt asteroids that have CCD spectra from 0.5 to $1.0 \mu \mathrm{m}$ that are distinctly similar to Vesta. Thirteen of these asteroids were found within the previously predicted Vesta family, while the other eight bridge the orbital space between Vesta and the Jovian $3: 1$ resonance (Figure 2). It appears very likely that all of these fragments were initially derived from Vesta through one or more impact excavations.

Binzel and $\mathrm{Xu}$ (1993) noticed that fifteen of these asteroids had spectra very similar to Vesta (and to eucrites) with absorption band centers near $0.94 \mu \mathrm{m}$ and classified them as V-types (Figure 3). However the remaining six asteroids had spectra that peaked more sharply at a shorter wavelength and showed a much deeper and less broad $0.90 \mu \mathrm{m}$ absorption band (Figure 4). These characteristics are suggestive of diogenites and these asteroids were classified with the letter $\mathrm{J}$ (mnemonic for the diogenite Johnstown). A principal component analysis (Burbine and $\mathrm{Xu}$ personal communication) shows that the $\mathrm{J}$ class does indeed separate from the $\mathrm{V}$ class. A true mineralogical distinction between the $\mathrm{V}$ and $\mathrm{J}$ classes requires near-infrared spectral data. Specifically, diogenites are distinguished from eucrites by the lack of a weak feldspar absorption band near $1.2 \mu \mathrm{m}$. 


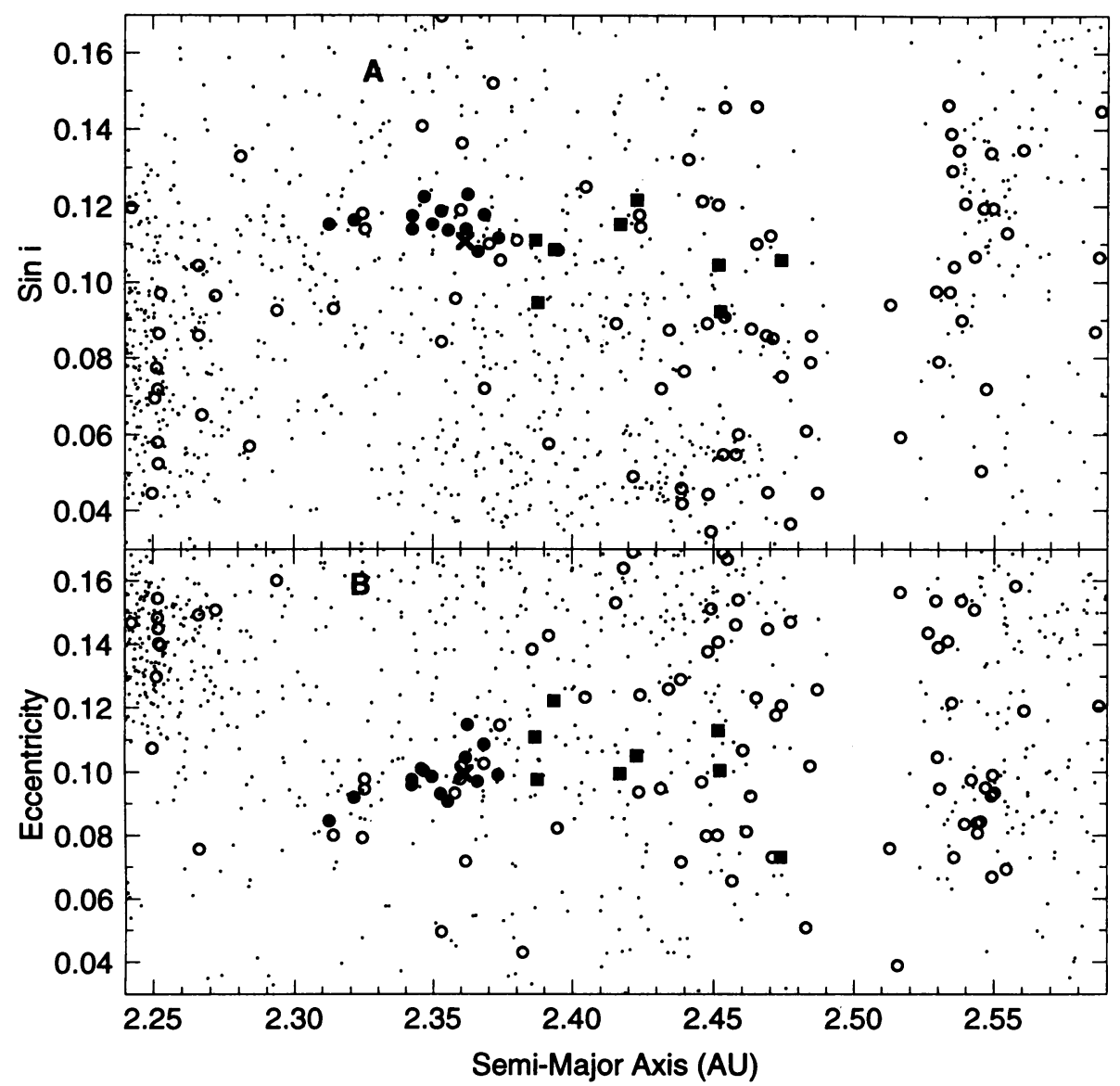

Fig. 2. Distribution of asteroids in proper orbital element space in the inner main belt for (A) semimajor axis versus $\sin i$ (inclination) and (B) semimajor axis versus eccentricity. Vesta's position is denoted by $\mathbf{X}$. The symbol $\bullet$ indicates the positions for the thirteen Vesta family asteroids that we have observed to display basaltic achondrite spectra. Positions for asteroids that we have also observed, but which do not have basaltic achondrite spectra are denoted by $\circ$. Eight additional discovered basaltic achondrite asteroids (filled squares), bridge the region between Vesta and the Jovian $3: 1$ resonance (2.50 AU). 


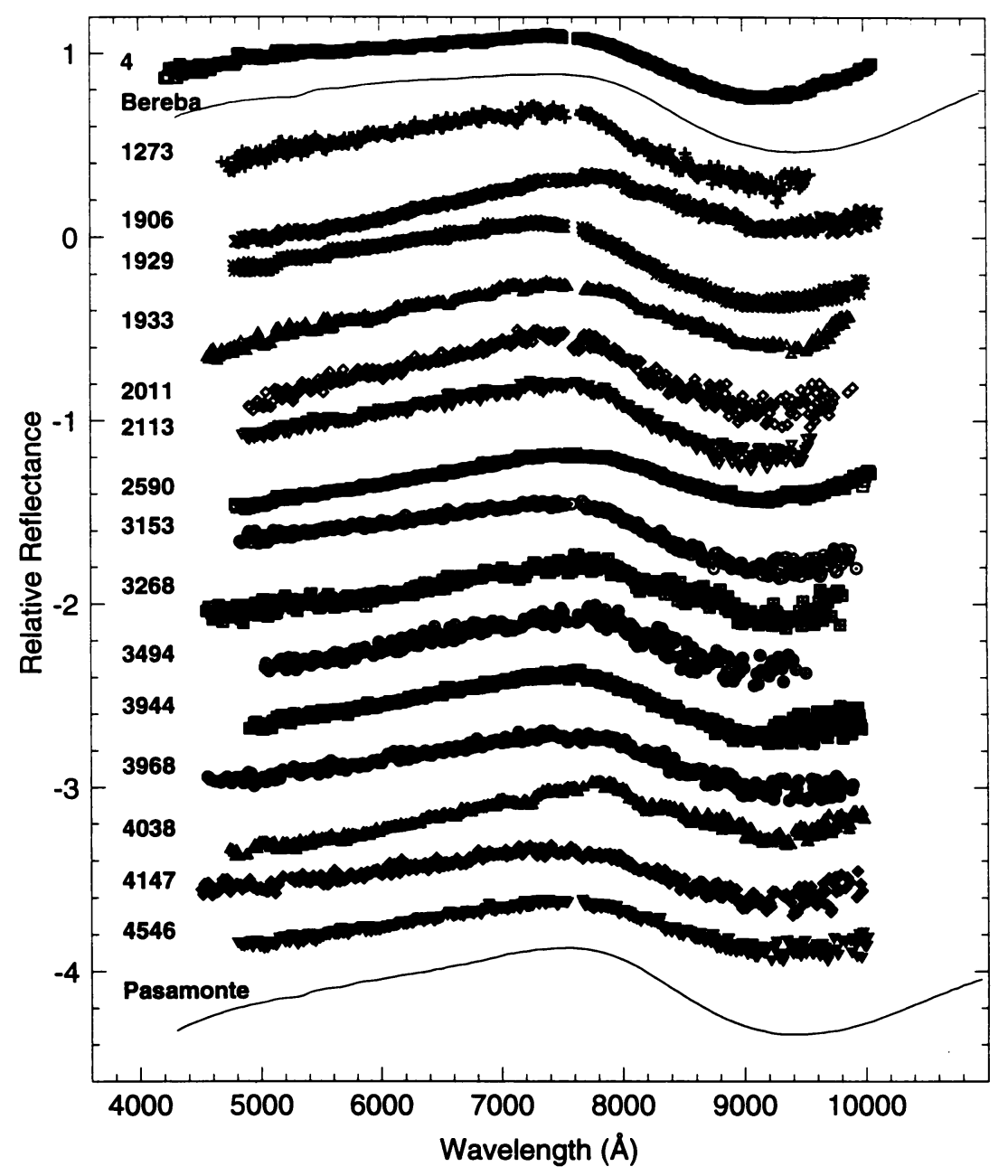

Fig. 3. Optical reflectance spectra for fifteen asteroids that display a deep absorption band near $0.9 \mu \mathrm{m}$, characteristic of basaltic achondrites. A spectrum of Vesta, obtained with the same instrumentation, and spectra of eucrites Bereba and Pasamonte (solid lines) (Gaffey 1976) are included for comparison. All spectra have been normalized to unity at $0.55 \mu \mathrm{m}$ and have been offset vertically for clarity. 


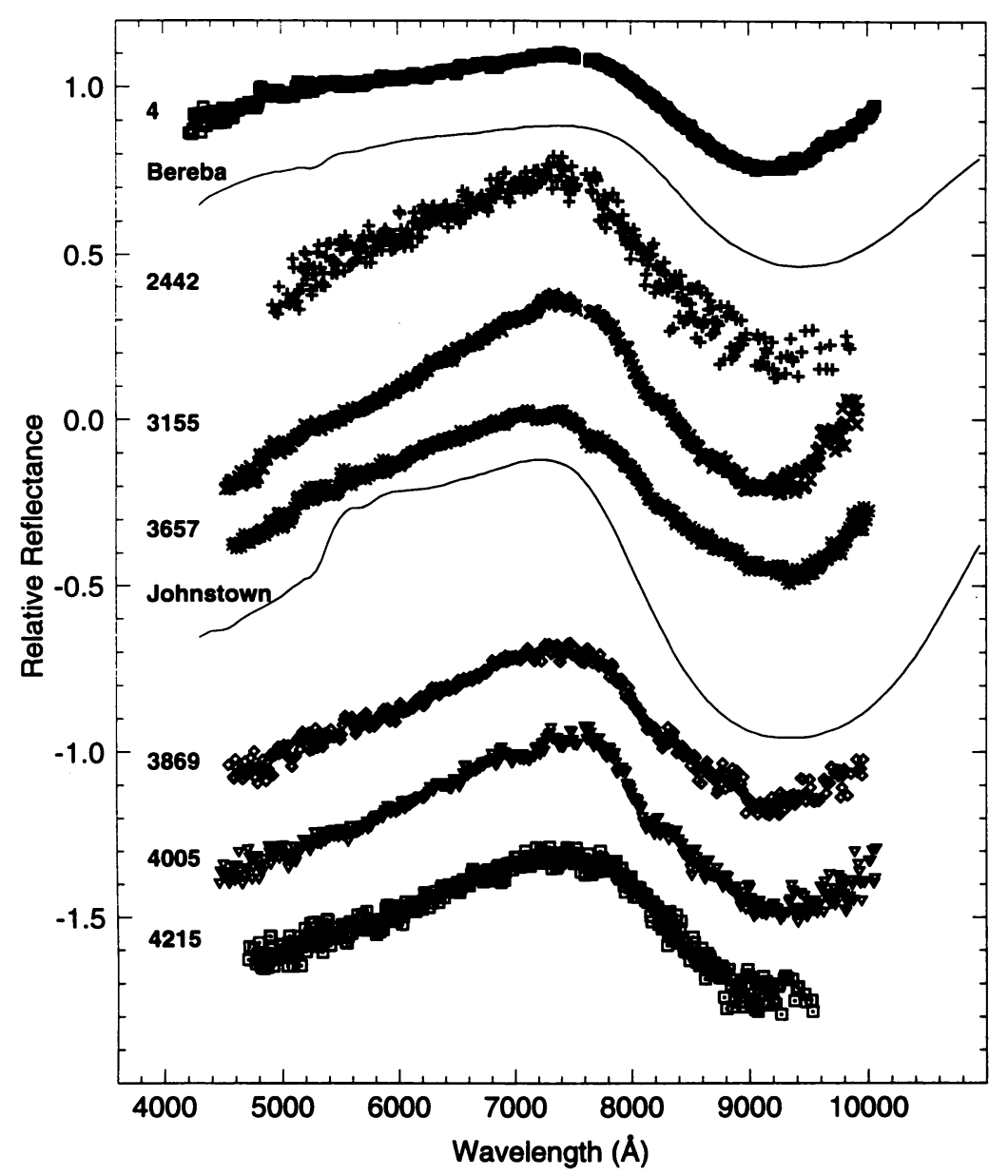

Fig. 4. Optical reflectance spectra for six additional basaltic achondrite main-belt asteroids. Vesta and the eucrite Bereba (Gaffey 1976) are included for comparison. Compared with eucrites, these asteroids show steeper slopes below $0.75 \mu \mathrm{m}$, sharper peaks and deeper $0.9 \mu \mathrm{m}$ absorption bands centered at a shorter wavelength. Their spectral properties are more analogous to the diogenites. A spectrum of the diogenite Johnstown (Gaffey 1976) is included for comparison. All spectra have been normalized to unity at $0.55 \mu \mathrm{m}$ and have been offset vertically for clarity. 


\section{Possible Main Belt Ordinary Chondrite Asteroid}

Also part of the CCD spectroscopic survey of Xu (1994) was the discovery of the spectrally anomalous characteristics of asteroid 3628 Božněmcová (diameter $\approx 7$ km) (Binzel et al. 1993). As shown in Figure 5A, Božněmcová has a CCD spectrum significantly different than the spectra from 0.5 to $1.0 \mu \mathrm{m}$ of all other known types of asteroids. The figure clearly shows that its spectrum is distinct from the $Q$ type asteroid 1862 Apollo. However the CCD spectrum of this asteroid appears very similar to the corresponding spectra of ordinary chondrites (Figure $5 B$ ). The only near-infrared data available for this object are from $\mathrm{JH}(1.2$ and $1.6 \mu \mathrm{m})$ photometry that appear roughly consistent with the ordinary chondrite spectra within the $1 \sigma$ error bars. Much higher resolution near-infrared data are needed to confirm the existence of an ordinary chondrite-like surface for this asteroid.

In addition to Božněmcová's spectral similarity to ordinary chondrites, it is also located near the Jovian 3: 1 resonance, a location which many researchers have predicted as an ordinary chondrite source region. It has been argued that many more ordinary chondrite-like objects should exist in this region and have not been seen only because of their small sizes (Bell et al. 1989). These postulated "small" ordinary chondrite asteroids could either be primordial objects or the relatively young fragments from larger precursor objects. However of the approximately 70 small asteroids (diameters $\leq 20 \mathrm{~km}$ ) observed in this region, only one of them had any spectral similarity to ordinary chondrites. Thus at present, the discovery of one possible ordinary chondrite asteroid does not resolve the problem for identifying the source(s) for this most common meteorite type.

\section{5145 Pholus}

Another spectrally anomalous asteroid is 5145 Pholus, which was discovered on 1992 January 9 just inside the orbit of Saturn (Scotti 1992). Pholus has a perihelion distance of 8.7 AU and an aphelion distance of 32.3 AU. CCD spectra from 0.5 to $1.0 \mu \mathrm{m}$ (Fink et al. 1992; Binzel 1992), BVRI colors (0.43 to $0.8 \mu \mathrm{m}$ ) (Mueller et al. 1992) and JHK colors (1.2, 1.6 and $2.2 \mu \mathrm{m}$ ) (Davies and Sykes 1992) show Pholus to be one of the reddest objects in the solar system. Its spectrum is unlike the spectrum of any known asteroid.

The spectrum of Pholus is best matched by the spectra of tholins (Cruikshank et al. 1991a), which are the nonvolatile residues produced by the irradiation of $\mathrm{C}$ bearing ices and gas mixtures. Due to its anomalous spectrum and distance from the sun, it has been postulated that Pholus is a "comet" that has yet to have "turned on" and started cometary activity (Fink et al. 1992, Mueller et al. 1992, Binzel 1992). Pholus has been tentatively classified as a Z-type asteroid (Mueller et al. 1992)

\section{Future Work}

The exciting new discoveries in asteroid spectroscopy and taxonomy within just the last few years appear to foretell of a promising and interesting future for the 1990s. 


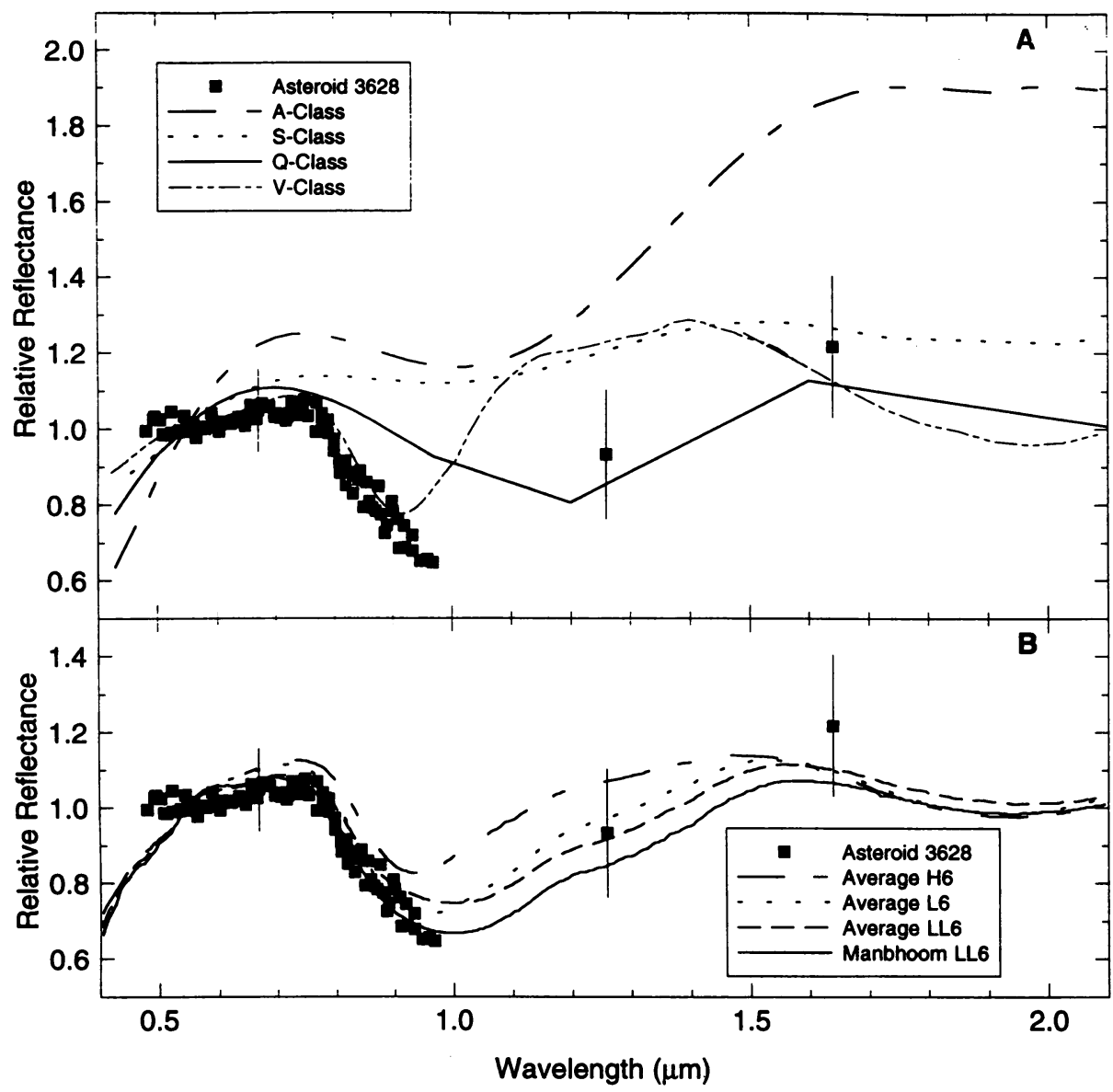

Fig. 5. Visible and near-infrared reflectance spectrum of the main-belt asteroid 3628 Božněmcová compared with (A) spectra of other asteroids and (B) spectra of ordinary chondrites. Error bars are affixed to the points denoting simultaneous measurements at $0.67,1.26$ and $1.64 \mu \mathrm{m}$. The spectral features of Božněmcová are presently unique among all measurements of main-belt asteroids. In comparison with meteorite spectra (B), the measurements of Božněmcová are most consistent with L6 and LL6 ordinary chondrites. All spectra have been normalized to unity at $0.55 \mu \mathrm{m}$. 
Continued directions toward surveying smaller asteroids and for obtaining spectra out into the near-infrared promise to illuminate many of the current problems in the field. Importantly the trend appears away from just assigning a taxonomic letter to individual asteroids. Instead, the trend is toward more thoroughly understanding the mineralogy of individual asteroids and making comparisons based on mineralogy. We are taking our first steps toward what might be called "asteroid geology."

\section{Acknowledgements}

This paper is dedicated to Roger Burns who passed away just as this paper was being completed. Roger's work on the spectroscopy of minerals is the basis for much of the mineralogical analyses of asteroids today. We would like to thank Shui $\mathrm{Xu}$ for the preparation of some of the figures. We would also like to thank Paolo Farinella and Vincenzo Zappalá for their reviews.

\section{References}

Barucci, M. A. and Lazzarin, M. : 1993, "Koronis family : Reflectance spectra of 243 Ida, 1442 Corvina and 2226 Cunitza." Planet. Space Sci, 41, 641-646.

Barucci, M. A., Capria, M. T., Coradini, A. and Fulchignoni, M. : 1987, "Classification of asteroids using G-mode analysis." Icarus, 72, 304-324.

Bell, J. F. : 1986, "Mineralogical evolution of meteorite parent bodies (abstract)." Lunar Planet. Sci., XVII, 985-986.

Bell, J. F. : 1988, "A probable asteroidal parent body for the $\mathrm{CV}$ or $\mathrm{CO}$ chondrites (abstract). Meteoritics, 23, 256-257.

Bell, J. F., Davis, D. R., Hartmann, W. K. and Gaffey, M. J. : 1989, "Asteroids : The big picture." In Asteroids II (R. P. Binzel, T. Gehrels and M. S. Matthews, Eds.), 921-945. University of Arizona Press.

Bell, J. F., Owensby, P. D., Hawke, B. R. and Gaffey, M. J. : 1988, "The 52-color asteroid survey : Final results and interpretation (abstract)." Lunar Planet. Sci., XIX, 57-58.

Binzel, R. P. : 1992, "The optical spectrum of 5145 Pholus." Icarus, 99, 238-240.

Binzel, R. P. and Xu, S. : 1990, "Asteroid 2965 Surikov : A piece of the missing mantle? (abstract)." Bull. Am. Astron. Soc., 22, 1115-1116.

Binzel, R. P. and Xu, S. : 1993, "Chips off of asteroid 4 Vesta : Evidence for the parent body of basaltic achondrite meteorites." Science, 260, 186-191.

Binzel, R. P., Xu, S., Bus, S. J., Skrutskie, M. F., Meyer, M. R., Knezek, P. and Barker, E. S. : 1993. "Discovery of a main-belt asteroid resembling ordinary chondrite meteorites." Science, 262, 1541-1543.

Burbine, T. H. and Bell, J. F. : 1993a, "Asteroid taxonomy : Problems and proposed solutions (abstract)." IAU Symposium 160 : Asteroids, Comets, Meteors 1993, 49.

Burbine, T. H. and Bell, J. F. : 1993b, "How diverse is the asteroid belt? (abstract)." Lunar Planet. Sci., XXIV, 223-224.

Burbine, T. H., Gaffey, M. J. and Bell, J. F. : 1992, "S-asteroids 387 Aquitania and 980 Anacostia : Possible fragments of the breakup of a spinel-bearing parent body with CO3/CV3 affinities." Meteoritics, 27, 424-434.

Clark, B. E. : 1993, "Spectral Reflectance Studies and Optical Surface Alteration in the Search for Links Between Meteorites and Asteroids." Ph. D. Thesis, University of Hawaii. 
Cloutis, E. A. and Gaffey, M. J., : 1993, "The constituent minerals in calcium-aluminum inclusions : Spectral reflectance properties and implications for $\mathrm{CO}$ carbonaceous chondrites and asteroids." Icarus, 105, 568-579.

Cloutis, E. A., Gaffey, M. J., Jackowski, T. L. and Reed, K. L. : 1986, "Calibration of phase abundance, composition, and particle size distribution for olivine-orthopyroxene mixtures from reflectance spectra." J. Geophys. Res., 91, 11,641-11,653.

Cruikshank, D. P., Allamandola, L. J., Hartmann, W. K., Tholen, D. J., Brown, R. H., Matthews, C. N. and Bell, J. F. : 1991a, "Solid C $\equiv N$ bearing material on outer solar system bodies." Icarus, 94, 345-353.

Cruikshank, D. P., Tholen, D. J., Hartmann, W. K., Bell, J. F. and Brown, R. H. : 1991b, "Three basaltic Earth-approaching asteroids and the source of the basaltic meteorites." Icarus, 89, 1-13.

Davies, J. K. and Sykes, M. V. : 1992, "(5145) 1992 AD." IAU Circular, 5480.

Farinella, P., Gonczi, R., Froeschlé, Ch. and Froeschlé, C. : 1993, "The injection of asteroid fragments into resonances." Icarus, 101, 174-187.

Feierberg, M. A. and Drake, M. A. : 1980, "The meteorite-asteroid connection : The infrared spectra of eucrites, shergottites, and Vesta." Science, 209, 805-807.

Feierberg, M. A., Larson, H. P., Fink, U. and Smith, H. A. : 1980, "Spectroscopic evidence for two achondrite parent bodies : Asteroids 349 Dembowska and 4 Vesta." journalGeochim. Cosmochim. Acta, 45, 971-981.

Fink, U., Hoffman, M., Grundy, W., Hicks, M. and Sears W. : 1992, "The steep red spectrum of 1992 AD : An asteroid covered with organic material?"Icarus, 97, 145149.

Gaffey, M. J. : 1976, "Spectral reflectance characteristics of the meteorite classes." $J$. Geophys. Res., 81, 905-920.

Gaffey, M. J. : 1983, "The asteroid (4) Vesta : Rotational spectral variations, surface material heterogeneity, and implications for the origin of the basaltic achondrites (abstract)." Lunar Planet. Sci., XIV, 231-232.

Gaffey, M. J. : 1984. "Rotational spectral variations of asteroid (8) Flora : Implications for the nature of the S-type asteroids and for the parent bodies of the ordinary chondrites." Icarus, 60, 83-114.

Gaffey, M. J., Bell, J. F., Brown, R. H., Burbine, T. H., Piatek, J. L., Reed, K . L. and Chaky, D. L. : 1993a, "Mineralogic variations within the S-type asteroid class." Icarus, 106, 573-602.

Gaffey, M. J., Bell, J. F. and Cruikshank, D. P. : 1989, "Reflectance spectroscopy and asteroid surface mineralogy." In Asteroids II (R. P. Binzel, T. Gehrels and M. S. Matthews, Eds.), 98-127. University of Arizona Press.

Gaffey, M. J., Burbine, T. H. and Binzel, R. P. : 1993b, "Asteroid spectroscopy : Progress and perspectives." Meteoritics, 28, 161-187.

Gaffey, M. J., Reed, K. L. and Kelley, M. S. : 1992, "Relationship of E-type Apollo asteroid 3103 (1982 BB) to the enstatite achondrite meteorites and the Hungaria asteroids." Icarus, 100, 95-109.

Howell, E. S., Merényi, E. and Lebofsky, L. A. : 1994, "Classification of asteroid spectra using a neural network." Jour. Geophys. Res., in press.

Jones, T. D., Lebofsky, L. A., Lewis, J. S. and Marley, M. S. : 1990, "The composition and origin of the $\mathrm{C}, \mathrm{P}$, and $\mathrm{D}$ asteroids : Water as a tracer of thermal evolution in the outer belt." Icarus, 88, 172-192.

Keil, K. : 1989, "Enstatite meteorites and their parent bodies." Meteoritics, 24, 195-208.

Luu, J. X. and Jewitt, D. C. : 1990, "Charge-coupled device spectra of asteroids. I. NearEarth and 3:1 resonance asteroids." Astron. J., 09, 1985-2011.

McCord, T. B., Adams, J. B. and Johnson, T. V. : 1970, "Asteroid Vesta : Spectral reflectivity and compositional implications." Science, 168, 1445-1447.

Meteorite Working Group, : 1993. Antarctic Meteorite Newsletter (R. Score and M. Lindstrom, Eds.), 16, 15. 
Mueller, B. E. A., Tholen, D. J., Hartmann, W. K. and Cruikshank, D. : 1992, "Extraordinary colors of asteroidal object (5145) 1992 AD." Icarus, 07, 150-154.

Ostro, S. J., Campbell, D. J. and Shapiro, I. I. : 1985, "Mainbelt asteroids : Dualpolarization radar observations." Science, 229, 442-446.

Pieters, C. M. and McFadden, L. A. : 1994, "Meteorite and asteroid reflectance spectroscopy : Clues to early solar system processes." Ann. Rev. of Earth and Plan. Science, in press.

Rivkin, A. S., Britt, D. T., Howell, E. S. and Lebofsky, L. A. : 1994, "Hydrated E-class and M-class asteroids (abstract)." Lunar Planet. Sci., XXV, in press.

Scotti J. V. : 1992, "1992 AD." IAU Circular, 5434.

Takeda, H., Saika, K., Otsuki, M. and Hiroi, T. : 1993, "A new Antarctic meteorite with chromite, orthopyroxene and metal with reference to a formation model of $\mathrm{S}$ asteroids (abstract)." Lunar Planet. Sci., XXIV, 1395-1396.

Tedesco, E. F., Matson, D. L., Veeder, G. J., Gradie, J. C. and Lebofsky, L. A. : 1989, "A three-parameter asteroid taxonomy." Astron. J., 97, 580-606.

Tholen, D. J. : 1984, "Asteroid Taxonomy from Cluster Analysis of Photometry." Ph. D. Thesis, University of Arizona.

Veeder, G. J., Hanner, M. S., Matson, D. L., Tedesco, E. F., Lebofsky, L. A. and Tokunaga, A. T. : 1989, "Radiometry of near-Earth asteroids." Astron. J., 07, 1211-1219.

Vilas, F. and McFadden, L. A. : 1993, "CCD reflectance spectra of selected asteroids. I. Presentation and data analysis considerations." Icarus, 100, 85-94.

Wetherill, G. W. : 1987, "Dynamical relations between asteroids, meteorites and ApolloAmor objects." Phil. Trans. R. Soc. Lond. A, s23, 323-337.

Wisniewski, W. Z. : 1987, "Photometry of six radar target asteroids.", Icarus, 90, 117122.

Xu, S. : 1994, "CCD Photometry and Spectroscopy of Small Main-Belt Asteroids." Ph. D. Thesis, Massachusetts Institute of Technology.

Xu, S., Binzel, R. P., Burbine, T. H. and Bus, S. J. : 1993, "Small main-belt asteroid spectroscopic survey (abstract)." Bull. Am. Astron. Soc., 25, 1135.

Zappalá, V., Cellino, P., Farinella, P. and Kneževic̀, Z. : 1990, "Asteroid families. 1. Identification by hierarchical clustering and reliability assessment." Astron. J., 100, 2030-2046.

Zellner, B., Leake, M., Morrison, D. and Williams, J. G. : 1977, "The E asteroids and the origin of the enstatite chondrites." Geochim. Cosmochim. Acta, 41, 1759-1767.

Zellner, B., Tholen, D. J. and Tedesco, E. F. : 1985, "The eight-color asteroid survey : Results for 589 minor planets.” Icarus, 61, 355-416. 